\title{
Rats Fail to Discriminate Quinine from Denatonium: Implications for the Neural Coding of Bitter-Tasting Compounds
}

\author{
Alan C. Spector and Stacy L. Kopka \\ Department of Psychology, University of Florida, Gainesville, Florida 32611
}

Recent molecular findings indicate that many different G-protein-coupled taste receptors that bind with "bittertasting" ligands are coexpressed in single taste receptor cells in taste buds, leading to the prediction that mammals can respond behaviorally to structurally diverse "bitter" tastants but cannot discriminate among them. However, recent in situ calcium-imaging findings imply that rat taste receptor cells are more narrowly tuned to respond to bitter-tasting compounds than had been predicted from molecular findings, suggesting that these animals can discriminate among these chemicals. Using an operant conditioning paradigm, we demonstrated that rats cannot discriminate between two structurally dissimilar bitter compounds, quinine hydrochloride and denatonium benzoate, despite the fact that these tastants are thought to stimulate different taste receptor cells. These rats were nonetheless able to show concentration-dependent avoidance responses to both compounds in brief-access tests and to discriminate among other taste stimuli, including quinine versus $\mathrm{KCl}$, denatonium versus $\mathrm{KCl}$, and $\mathrm{NaCl}$ versus $\mathrm{KCl}$. Importantly, the concentrations were varied in the discrimination tests to render intensity an irrelevant cue. We conclude that denatonium and quinine produce a unitary taste sensation, leaving open the likely possibility that other compounds fall into this class. Although a broader array of compounds needs to be tested, our findings lend support to the hypothesis that there is only one qualitative type of bitterness. These results also highlight the need to confirm predictions about the downstream properties of the gustatory system, or any sensory system, based on upstream molecular and biophysical events.

Key words: bitter taste; bitter discrimination; rat; psychophysics; quinine hydrochloride; denatonium benzoate; T2R receptor family; gustatory system
Natural selection has apparently favored animals endowed with the ability to avoid the ingestion of potentially harmful substances on the basis of taste. Many toxic compounds are reported as "bitter-tasting" by humans and are avoided by animals in a wide range of taxa (Garcia and Hankins, 1975; Glendinning, 1994; Delwiche et al., 2001). Recently, a family of G-protein-coupled taste receptors that interact with structurally diverse bitter-tasting chemical compounds was identified in mice and humans (Hoon et al., 1999; Adler et al., 2000; Chandrashekar et al., 2000; Matsunami et al., 2000). Although each type of receptor is thought to be relatively specific for its ligand, many appear to be coexpressed in subsets of taste receptor cells (TRCs). This latter finding has led researchers to hypothesize that mammals cannot discriminate between bitter-tasting stimuli, because a given TRC could potentially be stimulated by a wide variety of compounds (Adler et al., 2000; Chandrashekar et al., 2000).

However, a more recent study (Caicedo and Roper, 2001) demonstrated that many TRCs respond somewhat selectively to certain bitter tastants, at least with respect to five compounds that are commonly used in taste experiments (cycloheximide, quinine hydrochloride, denatonium benzoate, phenylthiocarbamide, and sucrose octaacetate). In their experiment, Caicedo and Roper

Received Oct. 3, 2001; revised Nov. 21, 2001; accepted Dec. 10, 2001.

This work was supported by Grant R01-DC01628 from the National Institute on Deafness and Other Communication Disorders. We gratefully acknowledge Ed Rodgers for his technical help during data collection. We thank John I. Glendinning, Timothy Hackenberg, Philip Teitelbaum, Shachar Eylam, and Laura Geran for providing comments on a draft of this manuscript. Portions of this work were presented at the 23rd Annual Meeting of the Association for Chemoreception Sciences in Sarasota, FL, April 2001.

Correspondence should be addressed to Dr. Alan C. Spector, Department of Psychology, P.O. Box 112250, University of Florida, Gainesville, FL 32611-2250. E-mail: spector@ufl.edu.

Copyright (ㄷ) 2002 Society for Neuroscience $\quad 0270-6474 / 02 / 221937-05 \$ 15.00 / 0$
(2001) measured intracellular $\mathrm{Ca}^{2+}$ responses in rat TRCs in situ. Of the 69 bitter-sensitive TRCs examined, $67 \%$ responded to only one of the five compounds, and $26 \%$ responded to only two of the five. According to these results, TRCs in situ appear to be more narrowly tuned, at least with respect to bitter-tasting compounds (cf. Gilbertson et al., 2001), than would be predicted from the molecular findings, raising the possibility that mammals can discriminate among these chemicals.

With the exception of a recent abstract (Lindsey and Breslin, 2001), we are not aware of any published studies that have directly assessed whether humans, ${ }^{a}$ rodents, or any vertebrate species can perceptually discriminate among purely bitter-tasting compounds. Such experiments are not as simple as they may initially appear. First, it is important to eliminate intensity cues in the experimental procedure. For example, humans can discriminate fructose from sucrose at isomolar concentrations, presumably on the basis of intensity, but there is a distinct pair of concentrations for which the taste of these two sugars is indistinguishable, thus leading researchers to conclude that these compounds produce a unitary taste sensation (Breslin et al., 1996). Second, if discriminability between two bitter-tasting compounds is displayed, it could be because one or both of the compounds stimulates receptor processes that lead to other qualitative taste sensations (e.g., sweetness). For example, humans report that saccharin has both bitter and sweet taste qualities (Bartoshuk, 1979; Schiffman et al., 1979). In fact, other potential cues, such as the temporal rise and decay of the sensation or its oral locus, could provide discriminable cues for compounds that have identical qualities.

${ }^{a}$ At the 23rd Annual Meeting of the Association for Chemoreception Sciences held in Sarasota, FL, in April 2001, Lindsey and Breslin (2001) reported that humans have difficulty discriminating among various bitter-tasting compounds when intensity cues are eliminated. 
Thus, it is perhaps more remarkable to find taste compounds among which animals cannot discriminate.

In this study, we chose two bitter compounds, quinine hydrochloride (found in tonic water) and denatonium benzoate (used to denature alcohol) and directly tested whether rats could discriminate between them behaviorally. Gene-deletion studies in mice implicate both compounds as acting, at least in part, through gustducin, a G-protein found specifically ${ }^{b}$ in TRCs (McLaughlin et al., 1992; Wong et al., 1996; Spielman, 1998). Importantly, denatonium and quinine are structurally dissimilar stimuli, and they stimulate different subsets (with some small degree of overlap) of rat TRCs based on measurements of changes in intracellular calcium concentration (Caicedo and Roper, 2001).

\section{MATERIALS AND METHODS}

An operant conditioning task was used in which intensity was rendered an irrelevant cue (Spector et al., 1996; St. John et al., 1997). We used a specially designed rodent taste-testing apparatus referred to as a gustometer (Spector et al., 1990). This device allowed us to deliver small volumes of taste stimuli and to measure immediate responses, thus increasing our confidence that the behavior was taste-guided. All stimuli were dissolved in distilled water and were prepared with reagent-grade chemicals. In both experiments reported here, water bottles were removed midday on Sunday and training and testing were conducted Monday through Friday; water bottles were replaced on the cages immediately after the Friday session of each week. Rats (Sprague Dawley; Charles River Laboratories, Wilmington, MA) received their daily allotment of fluid during these sessions. Any animal whose body weight dropped to $<85 \%$ of the ad libitum drinking value was given supplemental water. Laboratory chow (5001; PMI Nutrition International, Brentwood, MO) was available ad libitum in the home cage. The animals were housed individually in a room in which temperature, humidity, and lighting were automatically controlled. All manipulations were conducted during the light phase of the $12 \mathrm{hr}$ light/dark cycle.

First, it was necessary to establish ranges of concentrations for each compound that would produce comparable sensation magnitudes. Two groups ( $n=8$ per group) of thirsty rats were trained to lick an initially dry drinking spout to receive short periods $(5 \mathrm{sec})$ of access to water (St. John et al., 1994). After the animals were trained (two sessions), one group received various concentrations of denatonium benzoate (SigmaAldrich, St. Louis, MO) and water and the other group received various concentrations of quinine hydrochloride (Sigma-Aldrich) and water. The animals could initiate as many $5 \mathrm{sec}$ trials as possible within the $30 \mathrm{~min}$ sessions. A water-rinse trial was interposed between taste-stimulus trials (including water-stimulus trials) to minimize sensory adaptation from previous presentations. The stimuli were randomly presented without replacement within blocks of trials. There were three such testing sessions. Stimulus concentrations that produced comparable degrees of licking avoidance (taste-stimulus licks/water-stimulus licks) for the two compounds were chosen from the dynamic range of the concentrationresponse functions (Fig. 1, dashed lines and arrows) for use in the following discrimination experiment.

We subsequently attempted to train two different groups of naive thirsty rats to press one lever after sampling quinine hydrochloride and to press a different lever in response to a second taste stimulus. In the experimental group (group $2 ; n=8$ ), the second stimulus was denatonium benzoate; in the positive control group ( group $1 ; n=7^{c}$ ), the second stimulus was $\mathrm{KCl}$ (Fisher Scientific, Fair Lawn, NJ), a compound that we have shown previously rats can discriminate from quinine (St. John and Spector, 1998). Initially one midrange concentration of each taste stimulus was chosen for training (solution of $0.3 \mathrm{~mm}$ quinine hydrochloride, $0.879 \mathrm{~mm}$ denatonium benzoate, and $0.3 \mathrm{M} \mathrm{KCl}$ ).

Training consisted of five phases: alternation, random, discrimination training 1 (DT1), DT2, and DT3. During the alternation phase, a criterion number (CRIT) of correct lever presses to stimulus A was required before stimulus B was presented, and vice versa. The CRIT was systematically reduced across sessions. During the random phase, stimuli were randomly presented (without replacement) in successive blocks of two.

$\overline{{ }^{b}}$ There is evidence that the $\alpha$-subunit of gustducin is also expressed in brush cells in the epithelial lining of the stomach and intestine (Hofer, 1996).

${ }^{c}$ One rat in group 1 was removed from the experiment because of illness.

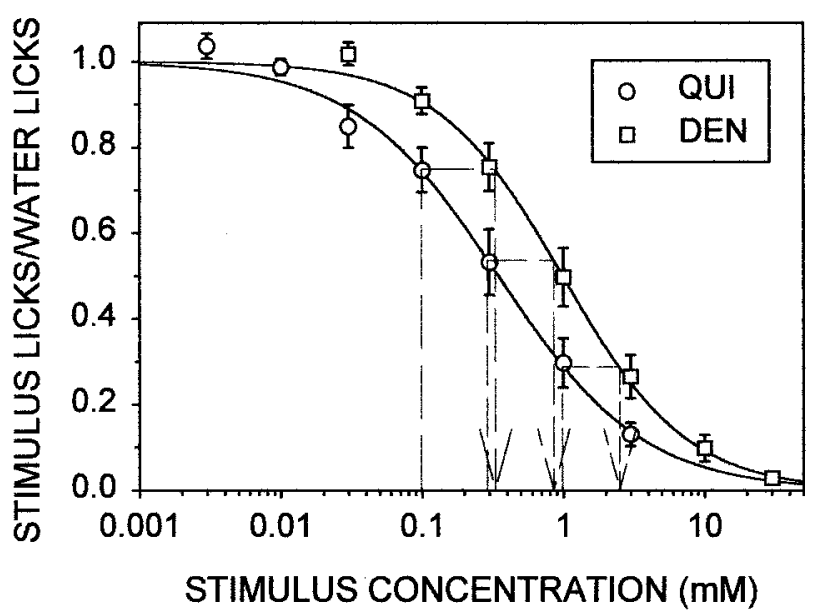

Figure 1. Dose-response functions illustrating the lick-suppressing effects of two bitter substances [quinine hydrochloride $(Q U I)$ and denatonium benzoate $(D E N)]$. Behavioral-suppression scores for each stimulus concentration were derived by taking the ratio of licks during stimulus trials relative to licks during water trials (only the last $3 \mathrm{sec}$ of the $5 \mathrm{sec}$ trials were used to eliminate the initial sampling response). Logistic functions were fit to the group mean data. In choosing matching stimulus concentrations to be used in the discrimination experiment, three quinine concentrations representing the dynamic range of lick suppression were first identified. Isoresponse concentrations of denatonium that suppressed licking to the same degree as the array of quinine concentrations were then derived (dashed lines and arrows).

During DT1, DT2, and DT3, session parameters were gradually changed to smaller sample and reinforcer volumes, a shorter decision period, and longer time-out periods. Importantly, the number of stimulus concentrations was increased from 1 to 3 for each stimulus to render intensity an irrelevant cue. Correct responses were rewarded with access to water, and incorrect responses or failure to respond within a limited period were punished with a time-out.

During the latter part of training and for the remainder of the experiment, the stimuli were presented in randomized blocks of six (i.e., three concentrations of each compound). The stimulus concentrations used during testing were: $0.1,0.3$, and $1.0 \mathrm{~mm}$ quinine hydrochloride; $0.352,0.879$, and $2.512 \mathrm{~mm}$ denatonium benzoate; $0.1,0.3$, and $1.0 \mathrm{M} \mathrm{KCl}$; and $0.1,0.3$, and $1.0 \mathrm{M} \mathrm{NaCl}$ (Fisher Scientific). The final parameters used in test sessions were: five licks or $3 \mathrm{sec}$ of stimulus sampling (whichever came first), a $5 \mathrm{sec}$ decision period (i.e., limited hold), 20 licks or $5 \mathrm{sec}$ of water reinforcement (whichever came first), $20 \mathrm{sec}$ of time-out, and an intertrial interval of $10 \mathrm{sec}$. As it turned out, the rats completed five licks of the sample spout for virtually every trial during testing, regardless of the stimulus. Performance on all trials with a lever press was averaged across all taste stimuli in a session and was tested against chance using one-sample $t$ tests for group data and the normal approximation to the binomial distribution for individual subject data (Brown and Hollander, 1977).

\section{RESULTS}

The rats were clearly responsive to quinine and denatonium and decreased their unconditioned licking in a concentrationdependent manner (Fig. 1). This allowed us to choose concentrations of both compounds for the discrimination experiment that produce comparable degrees of avoidance and therefore likely represent overlapping levels of intensity.

As shown in Figure $2 A$, the group 1 rats, which were trained to discriminate quinine from $\mathrm{KCl}$, acquired the task; during testing (Fig. $2 B$, far left) they performed with $\sim 90 \%$ accuracy [last day of testing: $t_{(6)}=25.4 ; p<0.001$; null hypothesis; $P$ (correct response $)=0.5(50 \%)]$. In striking contrast, the group 2 rats, which received quinine versus denatonium, never competently acquired the discrimination (Fig. $3 A$ ). The apparent discrimination during the early phases of training for group 2 was the result of a correc- 

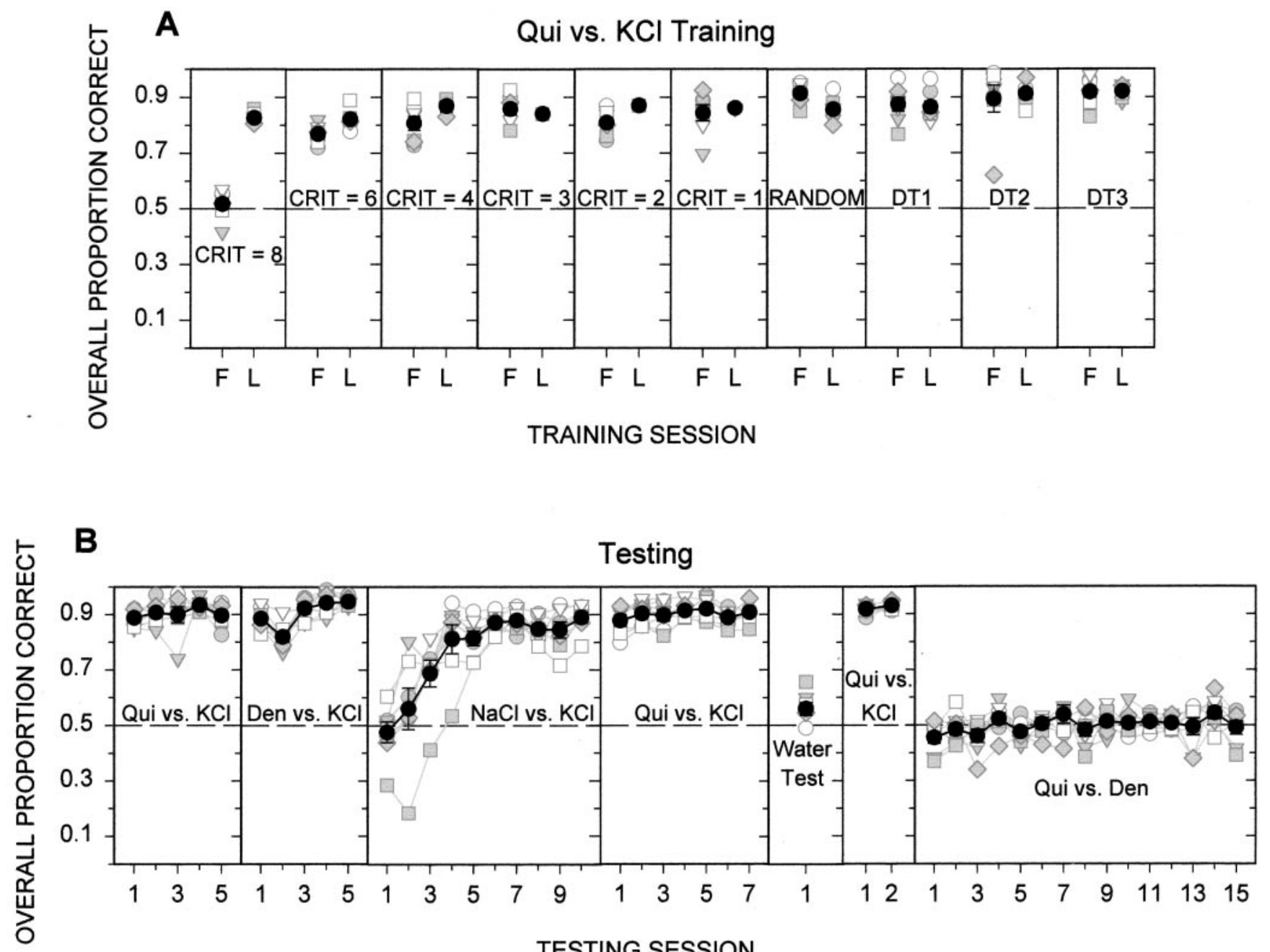

Figure 2. A, Individual animal ( gray and white symbols) and group mean ( \pm SE; black circles and bars) data for rats initially trained in quinine versus $\mathrm{KCl}$ discrimination (group 1) are plotted across training phases. Performance on all trials with a lever press is depicted averaged across all stimuli in a session. To conserve space, only the first $(F)$ and last $(L)$ day of each training phase are shown. During training, some rats were tested for only $1 \mathrm{~d}$ on a given criterion; thus, last day means represent only animals that were tested for $>1 \mathrm{~d}$ on the relevant criterion. It is clear that all of the animals learned the discrimination. $B$, Animals in group 1 were tested on a variety of taste discriminations, starting with the training stimuli (quinine vs $\mathrm{KCl}$ ) followed by denatonium versus $\mathrm{KCl}, \mathrm{NaCl}$ versus $\mathrm{KCl}$, quinine versus $\mathrm{KCl}$, water only, quinine versus $\mathrm{KCl}$, and finally quinine versus denatonium. Note that the substitution of denatonium for quinine had no effect on performance, whereas substitution of NaCl for denatonium substantially disrupted performance initially, but the animals eventually learned the discrimination. Performance was severely disrupted when only water was used, as it was when animals were tested on the quinine versus denatonium discrimination. All rats were included in all testing sessions. Because individual differences in performance were generally slight there is substantial overlap in symbols, making them difficult to discern in some cases. Chance performance equaled 0.5 .

tion procedure that was used in which the same compound was presented on successive trials until the animal had a criterion number of correct responses (i.e., alternation criterion). This alternation criterion was decreased during training until the stimuli were finally presented in random order (see Materials and Methods). Once the stimuli were presented randomly, these animals performed at chance levels (Fig. $3 A$, far right).

The group 1 animals, which had successfully been trained to discriminate quinine from $\mathrm{KCl}$, were then tested on a series of other discriminations (Fig. 2B). First, denatonium was substituted for quinine with virtually no disturbance of performance on the first session of the new discrimination (last session of $\mathrm{KCl}$ vs quinine compared with first session of denatonium vs quinine: $\left.t_{(6)}=0.515 ; p<0.625\right)$, a result consistent with the hypothesis that quinine and denatonium produce identical sensations. When denatonium was replaced with $\mathrm{NaCl}$, performance in these same animals dropped precipitously to chance during the first session $\left[t_{(6)}=-0.634 ; p=0.550\right.$; null hypothesis; $P$ (correct response) $=$ $0.5(50 \%)]$ and then progressively improved as the rats learned the new discrimination [last session: $t_{(6)}=20.53$; $p<0.001$; null hypothesis; $P$ (correct response) $=0.5(50 \%)]$. This latter manipulation demonstrates that changing one of the taste compounds in this discrimination task does have the potential to disrupt performance substantially, at least initially, yet replacing quinine with denatonium had no such effect.

When these animals were tested with only water as the stimulus (with one-half of the water reservoirs assigned to one lever and the other half assigned to the other lever), performance was severely impaired, confirming that rats were relying on the chemical nature of the stimuli to discriminate and not on extraneous cues associated with fluid delivery. Although the mean performance of the rats on the water control test was very close to 

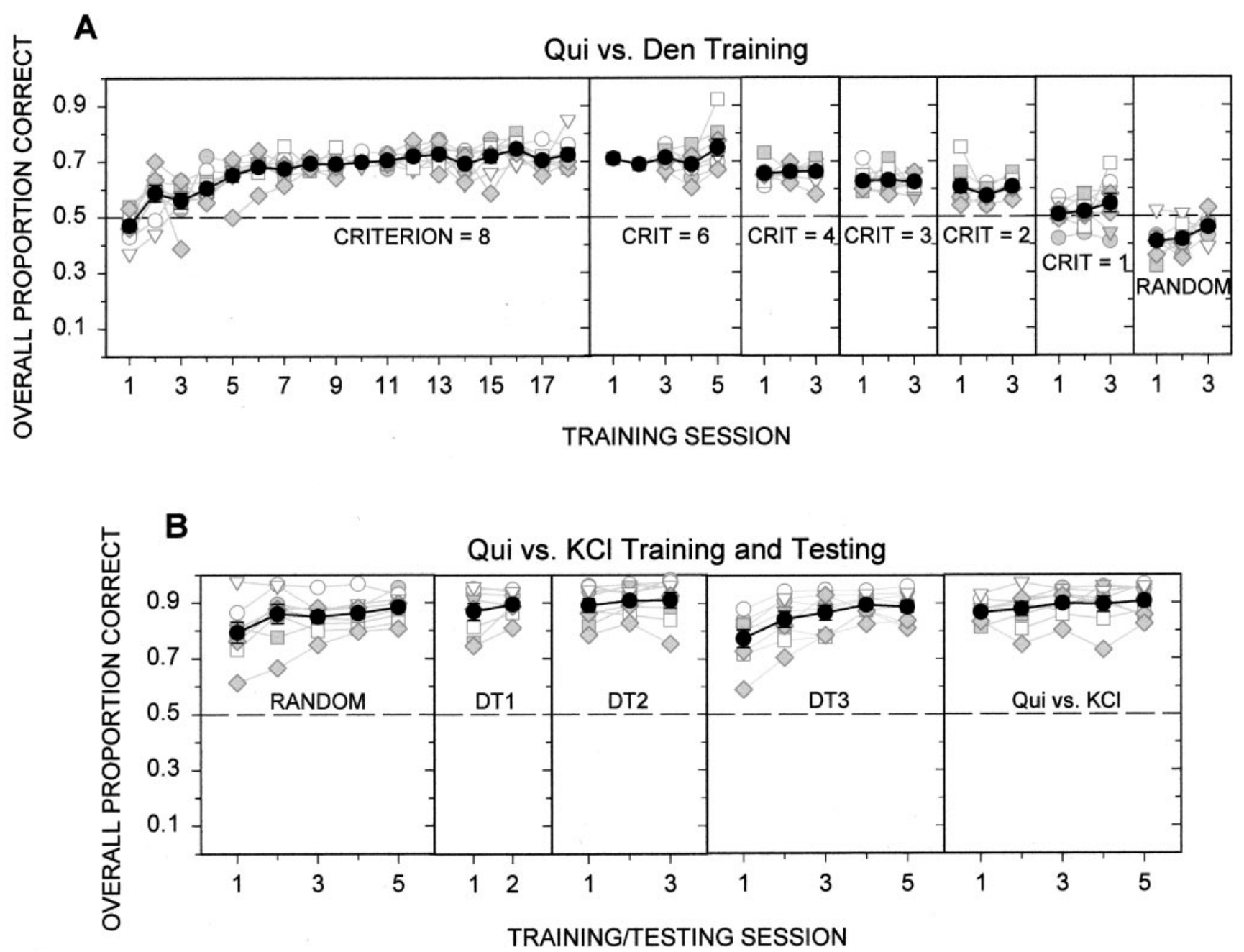

Figure 3. A, Individual animal ( gray and white symbols) and group mean ( \pm SE; black circles and bars) data for rats initially trained on a quinine versus denatonium discrimination (group 2) are plotted across training phases. Performance on all trials with a lever press is depicted averaged across all stimuli in a session. Once the alternation criterion during the correction procedure was lowered, performance progressively worsened until animals responded by chance when the stimuli were presented in randomized blocks. Because individual rats were tested for varying numbers of days at criteria 8 and 6 , group means for these two criteria do not necessarily represent all animals (e.g., sessions 1 and 2 of criterion 6). It is clear that all of the rats could not learn this taste discrimination. $B$, The animals in group 2 were then trained and tested on a quinine versus $\mathrm{KCl}$ discrimination. Only the final phases of training and testing are shown. It is clear that these rats were able to learn a quinine versus $\mathrm{KCl}$ discrimination. All rats were included in all testing sessions. Because individual differences in performance were generally slight there is substantial overlap in symbols, making them difficult to discern in some cases. Chance performance equaled 0.5.

chance, it was still significantly higher than 0.5 (mean $=0.56$; $\left.t_{(6)}=2.63 ; p=0.039\right)$. When scores from individual subjects were tested, only two of the seven rats had outlying scores significantly above chance, and these scores were very low $\left(R_{5}=0.60 ; R_{11}=\right.$ $0.66)$. However, these same two rats performed at chance levels when tested on the quinine versus denatonium discrimination (scores on last day of testing: $R_{5}=0.45 ; R_{11}=0.39$ ).

Finally, after the group 1 rats were successfully retested on the original quinine versus $\mathrm{KCl}$ task, denatonium was substituted for $\mathrm{KCl}$ (Fig. 2B). At this point, performance dropped to chance levels for the remainder of the experiment [last day: $t_{(6)}=-0.412 ; p=$ 0.695; null hypothesis; $P$ (correct response) $=0.5(50 \%)]$.

The group 2 rats, which had initially failed to discriminate quinine from denatonium, were then trained to discriminate quinine from $\mathrm{KCl}$ (Fig. 3B). Some of these rats acquired the discrimination at a slower rate than others, presumably because they were more disrupted by their early experience with the apparently impossible taste discrimination task. Nevertheless, all of the rats eventually learned to discriminate quinine from $\mathrm{KCl}$ and performed at nearly $90 \%$ accuracy on average, demonstrating that these animals had the capacity to learn a taste discrimination [last day: $t_{(6)}=19.4 ; p<0.001$; null hypothesis; $P$ (correct response $)=0.5(50 \%)]$.

\section{DISCUSSION}

These results strongly suggest that Sprague Dawley rats cannot distinguish perceptually between the respective tastes of denatonium benzoate and quinine hydrochloride, although these animals can respond to both compounds in a concentrationdependent manner. Because these are negative findings, we cannot conclusively rule out some discriminative capacity; however, in the context of the positive control manipulations, if rats can distinguish between these two bitter-tasting compounds they do so only poorly at best. It is worth noting that although explicit animal taste-discrimination experiments are scarce, quinine versus denatonium is the only taste discrimination, of which we are 
aware, that intact rats fail to express behaviorally. Rats have been shown behaviorally to discriminate $\mathrm{NaCl}$ from $\mathrm{KCl}$ (Brosvic and Hoey, 1990; Spector and Grill, 1992; St. John et al., 1995; Spector et al., 1996; Kopka et al., 2000), $\mathrm{NaCl}$ from $\mathrm{NH}_{4} \mathrm{Cl}$ (Geran et al., 2001), $\mathrm{KCl}$ from $\mathrm{NH}_{4} \mathrm{Cl}$ (Geran et al., 2001), quinine from $\mathrm{KCl}$ (St. John and Spector, 1998), sucrose from maltose (Spector et al., 1997), $\mathrm{NaCl}$ from sucrose (Morrison, 1967; Brosvic and Hoey, 1990), $\mathrm{NaCl}$ from sodium saccharin (Brosvic and Hoey, 1990), $\mathrm{NaCl}$ from quinine (Morrison, 1967), and $\mathrm{NaCl}$ from $\mathrm{HCl}$ (Morrison, 1967). Many of these discriminations were tested with the same procedure we used. Moreover, performance on many of these discrimination tasks was severely disrupted by manipulations of the peripheral gustatory system, demonstrating that the behavior was guided by taste cues. Caveats notwithstanding, these results lead to the conclusion that quinine hydrochloride and denatonium benzoate produce a unitary taste sensation, leaving open the likely possibility that other compounds fall into this class. Because many structurally diverse bitter-tasting compounds exist, it would be instructive to extend these findings by testing a broader array of such taste stimuli. Nevertheless, it is remarkable that two structurally dissimilar compounds described as bitter by humans, that are avoided by animals, and that appear to stimulate different TRCs were not discriminated by rats on the basis of taste as assessed behaviorally.

Caicedo and Roper (2001) examined 69 bitter-sensitive TRCs, 29 of which showed intracellular $\mathrm{Ca}^{2+}$ responses to quinine hydrochloride and/or denatonium benzoate. Of the 29 cells that responded to either compound, only 2 responded to both, suggesting a high discriminability at this level of the gustatory neuraxis, at least with regard to these two stimuli. However, our results indicate that these signals likely converge somewhere downstream in the flow of information. This could potentially occur at the level of gustatory afferent fibers (cf., Frank, 1991; Dahl et al., 1997) or in the brain. It is also interesting to note that in contrast to the dose-response functions representing changes in intracellular calcium concentration in TRCs (Caicedo and Roper, 2001), rats appear to be more responsive to quinine than to denatonium, as assessed behaviorally (Fig. 1). The differences between the two sets of findings noted above likely arise from the fact that the behavior represents the output of the entire gustatory system, whereas the biophysical findings are based on the initial stages of stimulus processing occurring in a subpopulation of TRCs in a restricted region of the tongue.

On the surface, our results appear to support the behavioral predictions arising from the molecular findings, indicating that chemically selective receptors that interact with structurally diverse bitter-tasting compounds are coexpressed on TRCs (Adler et al., 2000). However, the calcium-imaging findings (Caicedo and Roper, 2001) suggesting that TRCs have the potential to display reasonable discriminability among certain bitter-tasting compounds cannot be overlooked. Yet, according to the present results, such ability is not expressed in behavior. This complex set of findings from various levels of analysis highlights the importance of confirming predictions about the downstream properties of the gustatory system, or any sensory system, from upstream molecular and biophysical events (Spector, 2000).

\section{REFERENCES}

Adler E, Hoon MA, Mueller KL, Chandrashekar J, Ryba NJ, Zuker CS (2000) A novel family of mammalian taste receptors. Cell 100:693-702. Bartoshuk LM (1979) Bitter taste of saccharin related to the genetic ability to taste the bitter substance 6- $N$-propylthiouracil. Science 205:934-935.

Breslin PAS, Beauchamp GK, Pugh EN (1996) Monogeusia for fructose, glucose, sucrose, and maltose. Percept Psychophys 58:327-341.

Brosvic GM, Hoey NE (1990) Taste detection and discrimination performance of rats following selective desalivation. Physiol Behav 48:617-623.

Brown Jr BW, Hollander M (1977) Statistics: a biomedical introduction. New York: Wiley.

Caicedo A, Roper SD (2001) Taste receptor cells that discriminate between bitter stimuli. Science 291:1557-1560.

Chandrashekar J, Mueller KL, Hoon MA, Adler E, Feng L, Guo W, Zuker CS, Ryba NJ (2000) T2Rs function as bitter taste receptors. Cell 100:703-711.

Dahl M, Erickson RP, Simon SA (1997) Neural responses to bitter compounds in rats. Brain Res 756:22-34.

Delwiche JF, Buletic Z, Breslin PA (2001) Covariation in individuals' sensitivities to bitter compounds: evidence supporting multiple receptor/transduction mechanisms. Percept Psychophys 63:761-776.

Frank ME (1991) Taste-responsive neurons of the glossopharyngeal nerve of the rat. J Neurophysiol 65:1452-1463.

Garcia J, Hankins WG (1975) The evolution of bitter and the acquisition of toxiphobia. In: Olfaction and taste, Vol 5 (Denton DA, Coghlan JP, eds), pp 39-45. New York: Academic.

Geran LC, Garcea, M, Spector AC (2001) Discrimination between nonsodium salts is compromised by transection of the facial nerve. Appetite 35:287.

Gilbertson TA, Boughter Jr JD, Zhang H, Smith DV (2001) Distribution of gustatory sensitivities in rat taste cells: whole-cell responses to apical chemical stimulation. J Neurosci 21:4931-4941.

Glendinning JI (1994) Is the bitter rejection response always adaptive? Physiol Behav 56:1217-1227.

Hofer D (1996) Taste receptor-like cells in the rat gut identified by expression of alpha-gustducin. Proc Natl Acad Sci USA 93:6631-6634

Hoon MA, Adler E, Lindemeier J, Battey JF, Ryba NJ, Zuker CS (1999) Putative mammalian taste receptors: a class of taste-specific GPCRs with distinct topographic selectivity. Cell 96:541-551.

Kopka SL, Geran LC, Spector AC (2000) Functional status of the regenerated chorda tympani nerve as assessed in a salt taste discrimination task. Am J Physiol 278:R720-R731.

Lindsey AT, Breslin PA (2001) Taste matching among five bitter compounds: continuing studies. Chem Senses 26:1037.

Matsunami H, Montmayeur JP, Buck LB (2000) A family of candidate taste receptors in human and mouse. Nature 404:601-604.

McLaughlin SK, McKinnon PJ, Margolskee RF (1992) Gustducin is a taste-cell-specific $\mathrm{G}$ protein closely related to the transducins. Nature 357:563-569.

Morrison GR (1967) Behavioural response patterns to salt stimuli in the rat. Can J Psychol 21:141-152.

Schiffman SS, Reilly DA, Clark III TB (1979) Qualitative differences among sweeteners. Physiol Behav 23:1-9.

Spector AC (2000) Linking gustatory neurobiology to behavior in vertebrates. Neurosci Biobehav Rev 24:391-416.

Spector AC, Grill HJ (1992) Salt taste discrimination after bilateral section of the chorda tympani or glossopharyngeal nerves. Am J Physiol 263:R169-R176.

Spector AC, Andrews-Labenski J, Letterio FC (1990) A new gustometer for psychophysical taste testing in the rat. Physiol Behav 47:795-803.

Spector AC, Guagliardo NA, St John SJ (1996) Amiloride disrupts NaCl versus $\mathrm{KCl}$ discrimination performance: implications for salt taste coding in rats. J Neurosci 16:8115-8122.

Spector AC, Markison S, St John SJ, Garcea M (1997) Sucrose vs. maltose taste discrimination by rats depends on the input of the seventh cranial nerve. Am J Physiol 41:R1210-R1228.

Spielman AI (1998) Gustducin and its role in taste. J Dent Res 77:539-544.

St John SJ, Spector AC (1998) Behavioral discrimination between quinine and $\mathrm{KCl}$ is dependent on input from the seventh cranial nerve: implications for the functional roles of the gustatory nerves in rats. J Neurosci 18:4353-4362.

St John SJ, Garcea M, Spector AC (1994) Combined, but not single, gustatory nerve transection substantially alters taste-guided licking behavior to quinine in rats. Behav Neurosci 108:131-140.

St John SJ, Markison S, Spector AC (1995) Salt discriminability is related to number of regenerated taste buds after chorda tympani nerve section in rats. Am J Physiol 269:R141-R153.

St John SJ, Markison S, Guagliardo NA, Hackenberg TD, Spector AC (1997) Chorda tympani transection and selective desalivation differentially disrupt two-lever salt discrimination performance in rats. Behav Neurosci 111:450-459.

Wong GT, Gannon KS, Margolskee RF (1996) Transduction of bitter and sweet taste by gustducin. Nature 381:796-800. 